\title{
Systematic Screening of Behavioral Responses in Two Zebrafish Strains
}

\author{
Caroline Vignet, ${ }^{1}$ Marie-Laure Bégout, ${ }^{2}$ Samuel Péan, ${ }^{2}$ Laura Lyphout, ${ }^{2}$ \\ Didier Leguay, ${ }^{2}$ and Xavier Cousin ${ }^{1,3}$
}

\begin{abstract}
Wild-type (WT) zebrafish are commonly used in behavioral tests, but the term WT is not a precise description, and corresponds to many different strains (e.g., $\mathrm{AB}, \mathrm{TU}, \mathrm{WIK}$, and others). Previous studies compared the physiological, behavioral, or metabolic characteristics of different zebrafish strains (indigenous WT populations versus laboratory WT strains). AB and TU are widely used, but at least one study has demonstrated behavioral differences between them. To choose the most appropriate strain for our experiments, we systematically screened behavioral responses of $\mathrm{AB}$ and $\mathrm{TU}$ fish in several assays. We analyzed the locomotion activity and responses to a light/dark challenge in adults and larvae, and exploratory behavior and color conditioning in adults. Differences were observed for all tests, the strains displaying particular behavior depending on the tests. As larvae, TU displayed a wider activity range than $\mathrm{AB}$ larvae at the onset of locomotor behavior; as adults, TU were more reactive to sudden light transitions and recovered the swimming activity faster in T-maze or homebase release in novel tank tests, whereas $\mathrm{AB}$ fish had more contrasted circadian rhythms and performed better in color learning. Strain-specific behavior should be considered when designing experiments using behavior.
\end{abstract}

\section{Introduction}

Q UANTITATIVE BEHAVIOR ANALYSIS is now considered to be a good indicator of organism responses. Indeed, behavioral responses can be used to evaluate effects of a wide set of stimuli or stressors (e.g., chemicals, particular situations) in studies of pharmacology, toxicology, and ecotoxicology, and/ or in cognition or neurobiology. The use of model fish species, such as medaka or zebrafish, has developed exponentially over the last decade and their use in these contexts is now widely accepted. ${ }^{1-4}$ These model species have the advantage that they allow the combined analysis of an individual's physiology and molecular mechanisms. ${ }^{5-7}$ Furthermore, in the particular context of ecotoxicology, individual responses correspond to the interface between ecological factors and toxicity mechanisms, making individual behavior a particularly relevant and integrative indicator of the effects of pollutants. ${ }^{6,8-11}$

Most published articles report the use of wild-type (WT) strains of zebrafish. This general term includes numerous strains with variously long laboratory breeding histories (e.g., $\mathrm{AB}, \mathrm{TU}, \mathrm{TL}, \mathrm{WIK}$, and TM1) and also fish obtained from commercial suppliers (e.g., Scientific Hatcheries and petshops) or directly from the wild, such as Nadia, Gaighatta, and Bangladesh. ${ }^{12-19}$ Only genetically defined laboratory lines (including $\mathrm{AB}$ and $\mathrm{TU}$ ) will thereafter be named strains, while fish obtained from the wild or commercially available for which no information on genetics is available, will be, respectively, named wild or commercial populations. Several reports, mostly comparing laboratory strains to wild-caught and/or commercial populations, revealed differences in behaviors. ${ }^{12-31}$ Indeed, laboratory strains display evidence of domestication, including a loss of shoaling and antipredator behaviors (see, for example, the work of Robison's group ${ }^{16,18}$ ).

We report an analysis of the behavioral differences between two well-established laboratory strains, AB and TU. Our aim was to identify, which is more suitable for behavioral tests run in an ecotoxicology context. We monitored the spontaneous swimming activity and activity rhythm expression and recorded responses in challenging, exploration, and learning situations to detect possible differences between strains. Several well-established behavioral assays and age- and sizematched individuals from both strains were used.

Larvae and adult fish were subjected to four different behavioral challenges: the spontaneous swimming activity was measured over $48 \mathrm{~h}$ for adults and $72 \mathrm{~h}$ for larvae (from $4-7$ days postfertilization [dpf]). Reactivity was evaluated by applying a sudden dark change at the end of locomotion

\footnotetext{
${ }^{1}$ Laboratoire d'Ecotoxicologie, ${ }^{2}$ Laboratoire Ressources Halieutiques, Ifremer, L'Houmeau, France.

${ }^{3}$ INRA LPGP, Campus de Beaulieu, Rennes, France.
} 
recording for adults and in a specific test for $5 \mathrm{dpf}$ larvae. Exploration in a novel tank and responses in T-maze challenges and color learning in association with food presentation were studied in adults.

\section{Materials and Methods}

This study was conducted with the approval of the Animal Care Committee of France under the official license of M.-L. Bégout (17-010).

\section{Fish strains, rearing and larvae production}

We used two common laboratory strains: the AB strain (ZFIN ID: ZDB-GENO-960809-7) derived from individuals crossed in 1970 in Eugene; and the TU strain (ZFIN ID: ZDBGENO-990623-3), which was established in the 90's in Tubingen. These strains have been established in our laboratory for 6 years as large batches of individuals from the Amagen platform and Pasteur Institute fish facility. We used 3-12month-old adult and 4-7 dpf larvae. Adults were maintained at $27^{\circ} \mathrm{C}$ in a controlled 14 -h light/10-h dark (14:10) photoperiod in the same rack and were fed ad libitum twice a day with pellets (INICIO Plus 0.5; Biomar) between 9:00 and 9:30 in the morning and 16:30 and 17:30 in the afternoon and once with artemias (INVE) between 11:30 and 12:30. Eggs were obtained by random pairwise mating of zebrafish. One adult male and one female were placed together the evening before eggs were required in spawning boxes (AquaSchwarz). Eggs were collected in the morning and the fertilization rate assessed within $2 \mathrm{~h}$ of collection: only spawns with a rate above $80 \%$ were kept. At the same time, spawns were sorted to remove feces, and dead or unfertilized embryos. To provide as homogeneous and similar incubation conditions as possible, $50 \mathrm{em}-$ bryos from each spawn were transferred to an E3 medium. All analyses with larvae were performed using a mix of an equal number of embryos (10 to 40 embryos depending on assays) from five spawns and were repeated in three independents assays. Embryos and larvae were maintained at $28^{\circ} \mathrm{C}$ in Petri dishes in an incubator with the same photoperiod as adults. After hatching, chorions were removed manually and larvae were fed with artemias from $5 \mathrm{dpf}$ onward.

\section{Behavioral experiments}

Behavioral experiments were performed using 4 or $5 \mathrm{dpf}$ larvae, depending on the test (see below) and 3-12-month-old adult males in a dedicated room were kept at $27^{\circ} \mathrm{C} \pm 1^{\circ} \mathrm{C}$, with a 14:10 photoperiod synchronized with the rearing room so as to minimize unwanted correlated effects. Daylight started at 08:30 and there were no twilight transition periods.

\section{Forty-eight-hour swimming activity protocol and light/dark challenge in adults}

The purpose of these experiments was to monitor the circadian activity rhythm (48-h swimming activity) as well as photomotor responses used as an indicator of complex phenotypes of stress and anxiety. For each session, fish from each strain were placed randomly (to avoid tank position and session bias $)$ in twelve 3-L tanks $\left(24.5 \times 15 \times 13.5 \mathrm{~cm}\right.$, AquaBox ${ }^{\circledR} 3$; Aqua Schwarz $\mathrm{GmbH}$ ) filled with $1.5 \mathrm{~L}$ of system water. Tanks were placed in three rows with four tanks in each and were isolated from the neighboring tanks by opaque walls. We ran three sessions and the water was changed after each session. A camera was placed above the tanks that were on top of an infrared (IR) backlight device (IR floor $1 \times 1 \mathrm{~m}$; Noldus) to monitor horizontal movements. During the day, the room was lit with two halogen spotlights (Philips $80 \mathrm{~W}$ ) and IR lights were on. At night, the spotlights were turned off, and only IR light from the floor was used for recordings of fish movements. The fish were placed in their tanks at 17:00 the day before the experiment, for one night of acclimatization. Recording started the next day at $12: 30$ and lasted $48 \mathrm{~h}$. At the end of the $48-\mathrm{h}$ recording, the fish activity was further recorded for $1 \mathrm{~h}$ (light on1 ), then they were challenged with a sudden darkness (15 min, light off) and video recordings continued for 1 h30 (light on-2).

For both protocols, 18 fish of each strain were recorded and challenged and the dependent variable measured was the swimming path length (distance travelled, $\mathrm{cm}$ ).

\section{Seventy-two-hour swimming activity setup in larvae}

This method was used to monitor the onset of the swimming activity as well as the expression of the circadian activity rhythm in larvae. To obtain a higher level of activity, we have selected 24-well plates rather than higher throughput 48- or 96-well plates after Padilla et al. ${ }^{41}$

Three dpf larvae (10 AB and $10 \mathrm{TU})$ were individually transferred by the end of the day, ca. 17:00, to the wells of a 24well plate (Krystal 24, opaque [white] clear bottom microplate), where they were arranged in a mixed design (larvae from both strains were studied at the same time to avoid any trial effect) and visually isolated from each other. The four corner wells were left empty because video acquisition suffered distortion. The 24-well plates were kept overnight in an incubator with a cover placed on top of the plate to reduce evaporation. The following day $(4 \mathrm{dpf}), 2 \mathrm{~h}$ before the challenge, the well plate was transferred to the video acquisition room and placed on top of a size-matched IR floor, which allowed the larvae to be filmed under both light and dark conditions. A three-way switch permitted filming in the light or dark with constant IR lighting. The entire apparatus was enclosed within a lightproof and temperature-controlled box (hereafter called the "larvae-box"). The recording of the larval swimming activity started at 12:00 and was continued for $72 \mathrm{~h}$. The dependent variable measured was the distance travelled $(\mathrm{cm})$, and was recorded for 30 larvae per strain.

\section{Light/dark challenge in 5 dpf larvae}

This challenge was used to monitor the photomotor response. In larvae, it is a classically used test in a wide range of studies from basic research to applied drug screening. The same protocol and the same material were used for this experiment except that larvae were acclimatized in the 24-well plates at $4 \mathrm{dpf}$ and tested at $5 \mathrm{dpf}$. These experiments were conducted between 13:00 and 18:00 h, corresponding to the most stable activity period for zebrafish larvae. ${ }^{9}$ Larvae were challenged in a dark context (5 min light off) under two conditions (with or without an acclimatization period).

- With acclimatization in the larvae-box (protocol 1, P1): the plates were transferred to the video acquisition room at 11:30 and larvae were acclimatized in the larvae-box for $2 \mathrm{~h}$ before tests began at 13:30. Video recordings were made over three periods: before ( $2 \mathrm{~h}$, light on- 1$)$, 
during (5 min, light off), and after light off $(2 \mathrm{~h}$, light on-2). The experiment was stopped at 17:35 and only one plate per day was recorded.

- Without acclimatization in the larvae-box (Protocol 2, P2): the experiment was again started at 11:30, but one plate was transferred every $20 \mathrm{~min}$ to the room and left for $2 \mathrm{~h}$ on a back-lighted Plexiglas ${ }^{\circledR}$ plate beside the larvae-box. Recordings started at 13:30 for the first plate after its transfer to the larvae-box. Video recordings were made over three 5-min periods: before ( $5 \mathrm{~min}$, light on-1), during (5 min, light off), and after light off (5 min, light on-2) and up to 10 plates per day were processed, compatible with a high-throughput challenge procedure.

The dependent variable measured was the distance travelled $(\mathrm{cm})$ and was recorded for 40 (P1) or 100 (P2) larvae per strain.

\section{Exploration in a novel environment in adults}

In addition to providing information on the exploratory ability of the fish, the swimming characteristics recorded during exploration are giving indications as to how fish cope with this novel environment. Two experimental models were used: a T-maze and a novel tank challenge. The T-maze was slightly adapted from ${ }^{32}$ and comprised (1) a shallow area, with a water depth of $5 \mathrm{~cm}$, composed of the base of the $T$ (46 cm long), and two arms (one leading to the deep area and one in the opposite direction (total length of these two arms was $66 \mathrm{~cm}$ ) and (2) a deep area, which was $15 \mathrm{~cm}$ deep $(10 \mathrm{~cm}$ water depth), $23 \mathrm{~cm}$ wide, and $23 \mathrm{~cm}$ long, containing marbles and plastic grass and was considered to be a favorable zone. Fish were individually acclimatized in a 1-L aquarium at 16:00 on day 1 and challenged on day 2 between 8:00 and 18:00. Fish were placed in the start area of the maze and swimming characteristics recorded for $5 \mathrm{~min}$. Twenty fish were challenged per strain and the dependent variables measured were the latency to first exit from the start area (s), the time spent in each area $(\mathrm{s})$, and the distance travelled $(\mathrm{cm})$ per minute.

The novel tank challenge was performed after $2 \mathrm{~h}$ of acclimatization in the room in a 1-L aquarium. Fish were transferred to a novel tank (trapezoid 1.5-L tank; aquatic habitats; sizes in $\mathrm{cm}$ : height $15.2 \times$ width $7.1 \times$ length 27.9 at the top and 22.5 at the bottom) and filmed for $6 \mathrm{~min}$ as previously described. ${ }^{33}$ Twelve fish were challenged per strain. For space occupancy analysis, tanks were separated into two zones: the top zone, including one third of the volume and the bottom zone, including two-thirds, the dependent variable measured was the time spent in each zone per minute.

\section{Color learning by adult zebrafish}

The purpose of this test was to evaluate conditioned learning in both strains by using a color preference test. In the fish rearing facility, six 20-L glass aquariums on one rack were selected; three housed $15 \mathrm{TU}$ adult males and three housed 15 $\mathrm{AB}$ adult males. For each strain, two tanks were subjected to colored light conditioning; the third tank was a control. Fish were fed normally with pellets according to the schedule described previously. For conditioned tanks, a green light, located in front of the tank was switched on during $10 \mathrm{~s}$ and $2 \mathrm{~mL}$ of artemias was distributed $2 \mathrm{~s}$ after light-on. Distribution and conditioning were made once a day and the artemias concentration was high enough to allow all fish in the tank to have their share. This operation was repeated daily for 15 days. The experiments were performed on days 16 to 20 .

Fish were tested in a behavioral apparatus inspired by Risner $e t$ al $^{34}$ and hereafter called the "color-test box". It was $45 \mathrm{~cm}$ long and $32 \mathrm{~cm}$ wide and composed of two main areas: the home area and chamber area. Its walls were made of white opaque Komate ${ }^{\circledR}$ and its bottom was made of transparent Plexiglas ${ }^{\circledR}$. It was placed on a size-matched IR floor lit by 2 IR spot lights (28 LEDs-12 Lux, Ref. 203900; SimRadio). The home area was $32 \mathrm{~cm}$ wide and $30 \mathrm{~cm}$ long and had black masking material on its walls to prevent light scatter. The chamber area was separated into three open chambers by opaque Komatex dividers so the visual stimulus could be presented to each chamber independently. The chamber area was $15 \mathrm{~cm}$ wide and $32 \mathrm{~cm}$ long and each of the three chambers was $15 \mathrm{~cm}$ wide and $10 \mathrm{~cm}$ long. The back wall of each individual chamber was made of diffusing Plexiglas. Three different LED spots with each a on/off switch and a remote control for choosing the light color, either green or blue (LED RGB 3W), were positioned behind the back wall. The colors were chosen according to Mueller and Neuhauss. ${ }^{35}$ The colortest box was filled with $12 \mathrm{~L}$ of system water and placed in a dedicated room kept at $27^{\circ} \mathrm{C}$ and lighted with one halogen spotlight (Philips $80 \mathrm{~W}$ ). A camera was installed above the box (in top view) and allowed recording the fish in all areas.

Fish were subjected to two tests at the time of the day corresponding to conditioning (11:30-12:30). In the first test (test 1), they were gently individually placed in the start zone of the home area and recorded until the end of the test. After $2 \mathrm{~min}$, one randomly picked chamber was lit in green for $30 \mathrm{~s}$, and the two remaining chambers remained under ambient light. In the second test (test 2), the same protocol was used except that when the green light was on, an additional randomly picked chamber was lit in blue for the same 30-s duration. The variable measured was the time spent in each area and chamber; 15 control fish and 30 conditioned fish of each strain were challenged.

\section{Data recording and analysis}

Videos for the swimming activity, T-maze exploration, and novel tank experiments with adults were recorded with an analogue camera ICD-48E (Ikegami) and 2.7-13.5-mm lens (Fujinon) linked to a PC with an acquisition card and EthoVision XT software (Noldus). Videos for the larvae locomotion and adults color learning experiments were recorded using a digital DMK31AU03 camera (The Imaging Sources) and 1.4-12.5-mm lens (Fujinon) using IC-Capture software (The Imaging Sources).

For all experiments, EthoVision software was used for track extraction and analysis. Data were acquired by EthoVision at 25 frames per second, and variables (distance travelled, time spent in each area) were nested for further treatments every 30-min for locomotion in adults (48-h) and in larvae (72-h), or every 1-min for adults and every 30-s period for larvae for light/dark challenge experiments.

\section{Statistical analysis}

The results reported in text and all figures are mean \pm SEM. All fish used in these experiments (except the ones in the color learning test) were anesthetized in benzocaïne (SigmaAldrich; $50 \mathrm{mg} / \mathrm{L}$ in water from a $100 \mathrm{~g} / \mathrm{L}$ stock solution in ethanol) at the end of the challenge and were measured for 
mass and length, differences between strains were tested using one-way ANOVA. In case AB and TU differed in mass and/or length, the effects of differences were resolved by comparing all variables relating to the swimming activity in a repeated measures analysis of covariance, ANCOVA. Fish strain ( $\mathrm{AB}$ and $\mathrm{TU}$ ) was taken as a between-subject factor and body mass or length as a covariate and showed no significant interaction with strain whatever the test used.

For the 48- or 72-h swimming activity experiments, transition periods induced an exacerbated pattern; therefore, it was necessary to analyze these 30-min light off and light on periods immediately following the light change separately. Remaining day and night periods were divided into blocks of six or seven 30-min periods. Within each period, ANOVA tests were used to compare the distances travelled between the different strains. The distance travelled was also compared between daytime and nighttime, excluding the transitional half-hour periods, using ANOVA. For light/dark challenge experiments, distances travelled were compared over three periods of $15 \mathrm{~min}$ each for adults and $5 \mathrm{~min}$ each for larvae: before (light on-1), during (light off), and after light off (light on-2), in both cases, repeatedmeasure ANOVA (RM-ANOVA) was used followed by Newman-Keuls post hoc tests. For T-maze tests, latency to first exit of the start area, the time spent by fish in the three areas of interest (shallow, start, and deep sections), occupation of different zones, and the distance travelled were compared between strains with ANOVA tests. The same statistical tests were used to compare occupation of the upper and bottom zones in the novel tank test. For both assays, a discretization per minute was performed to analyze any temporal evolution of the distance travelled (T-maze) and top zone occupancy (novel tank) using RM-ANOVA followed by Newman-Keuls post hoc tests. Finally, the time spent in the different chambers during color challenge was compared between strains using ANOVA. All statistical analyses were performed with Statistica 9.0 software (Statsoft) and the significance of results was ascertained at $p<0.05$.

\section{Results}

\section{Forty-eight-hour swimming activity in adults and 72- $h$ swimming activity in larvae: spontaneous activity}

In 6-month adults and for both strains, mass were homogeneous $\left(\mathrm{AB}=178.4 \pm 6.8 \mathrm{mg}, \mathrm{TU}=160.7 \pm 6.4 \mathrm{mg}, \mathrm{F}_{(1,36)}=3.58\right.$, $p=0.066)$, but length differed $(\mathrm{AB}=2.37 \pm 0.03 \mathrm{~cm}, \mathrm{TU}=2.29 \pm$ $\left.0.02 \mathrm{~cm}, \mathrm{~F}_{(1,36)}=5.06, p=0.031\right)$, diurnal activity, evaluated as distance travelled, was the highest in the morning, and then decreased to a minimum at midday before increasing again in the afternoon. The nocturnal activity followed a similar U-shape
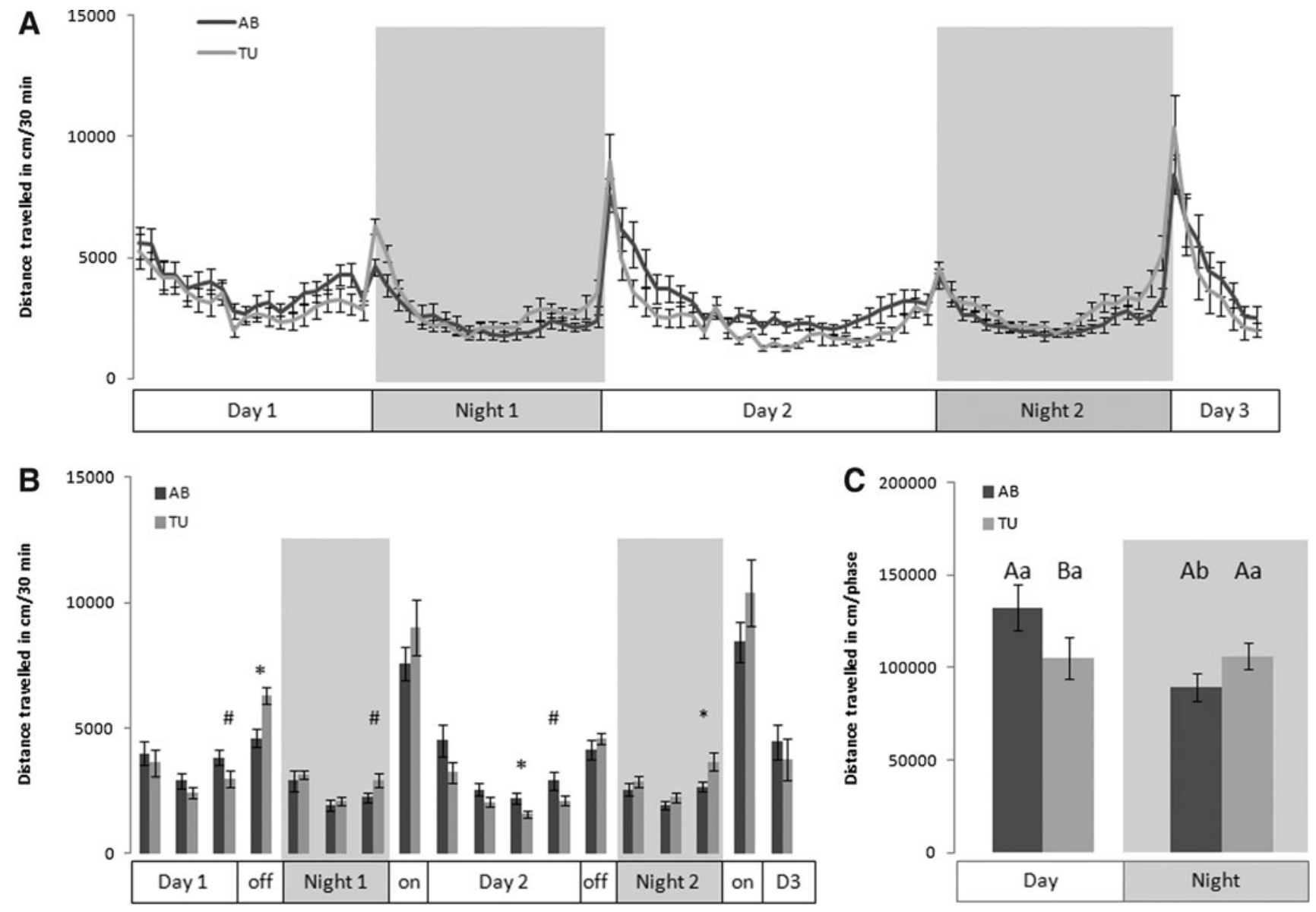

FIG. 1. Daily spontaneous activity of adult zebrafish. (A) Locomotor activity nested per 30-min period. (B) Cumulated locomotor activity per block of six or seven 30-min periods. The grayscale background indicates the light status/period of the day. (C) Locomotor activity during daytime and nighttime periods. [Mean \pm SEM; ${ }^{*} p<0.05$; ${ }^{\#} p<0.1$; in (C), upper case letters indicate significant differences between strains within periods and lower case letters indicate significant differences between periods within strains; $n=18$ per strain]. 
although the distance moved was shorter than during the daytime (Fig. 1A). Differences were observed for last daytime and nighttime blocks with $\mathrm{AB}$ fish being more active than $\mathrm{TU}$ fish at the end of the day and the opposite at the end of the night (Fig. $1 B)$. The distance travelled by $\mathrm{AB}$ fish during the day was greater than that by TU fish (ANOVA $\mathrm{F}_{(1,35)}=5.87 ; p=0.021$; Fig.1C), such that the cumulated distances travelled indicated that $\mathrm{AB}$ fish were diurnal, and that this was not the case for TU fish.

For both strains, the larval activity increased during $4 \mathrm{dpf}$, reached a plateau at $5 \mathrm{dpf}$, and then decreased until the end of the experiment (Fig. 2A). During night periods, the activity decreased to a minimum similar for all three nights and both strains. The activity of TU larvae was higher compared with $\mathrm{AB}$ larvae for almost all daytime periods. $\mathrm{AB}$ larvae became more active than TU larvae during most night periods after $5 \mathrm{dpf}$ (Fig. 2B). The cumulated distance travelled for all day and all night periods indicated that larvae of both strains were clearly diurnal, and confirmed that TU larvae were both more active than $\mathrm{AB}$ larvae during the day $\left(\mathrm{ANOVA}_{(1,60)}=31.36\right.$; $p<0.001)$ and displayed a more contrasted activity between day and night (Fig. 2C).

\section{Light/dark challenge}

Light/dark challenge was applied to both adults (after the 48-h activity recording; Fig. 3 ) and larvae (at 5 dpf; Fig. 4).
In 6-month-old adults (same fish as above) and for both strains, the light change produced an immediate and sustained increase in activity that lasted throughout the following dark period. The subsequent light on- 2 elicited an additional increase of activity that decreased rapidly afterward (Fig. 3A). For both strains, the various periods appeared significantly different from each other except in the case of light off/light on-2 comparison for $A B$ strain (Fig. 3B). The only difference between strains was during the dark period, when TU fish showed more activity than AB fish (RM$\left.\operatorname{ANOVA~}_{(3,18)}=5.34 ; p=0.004\right)$.

Two protocols were used for larvae: with (P1) or without (P2) an acclimatization period in the larvae-box.

For the duration of the P1 test as a whole, TU larvae were less active than $\mathrm{AB}$ larvae. For both strains, light off produced a synchronization of activity and larvae started with similar activity levels. The activity of $\mathrm{AB}$ larvae progressively increased during light on-2 and reached a plateau identical to that during light on-1 period $\sim 30$ min after light off; the activity increase for TU larvae during the corresponding period was limited (Fig. 4A). When considering periods of equivalent duration (Fig. 4B), ANOVA analysis indicated that for both strains, the activity during the dark period was significantly higher than the preceding and following light periods (ANOVA $\mathrm{F}_{(2,40)}=11.98 ; p<0.001$ for $\mathrm{AB}$ larvae and $\mathrm{F}_{(2,40)}=12.65 ; p<0.001$ for TU larvae). This analysis also
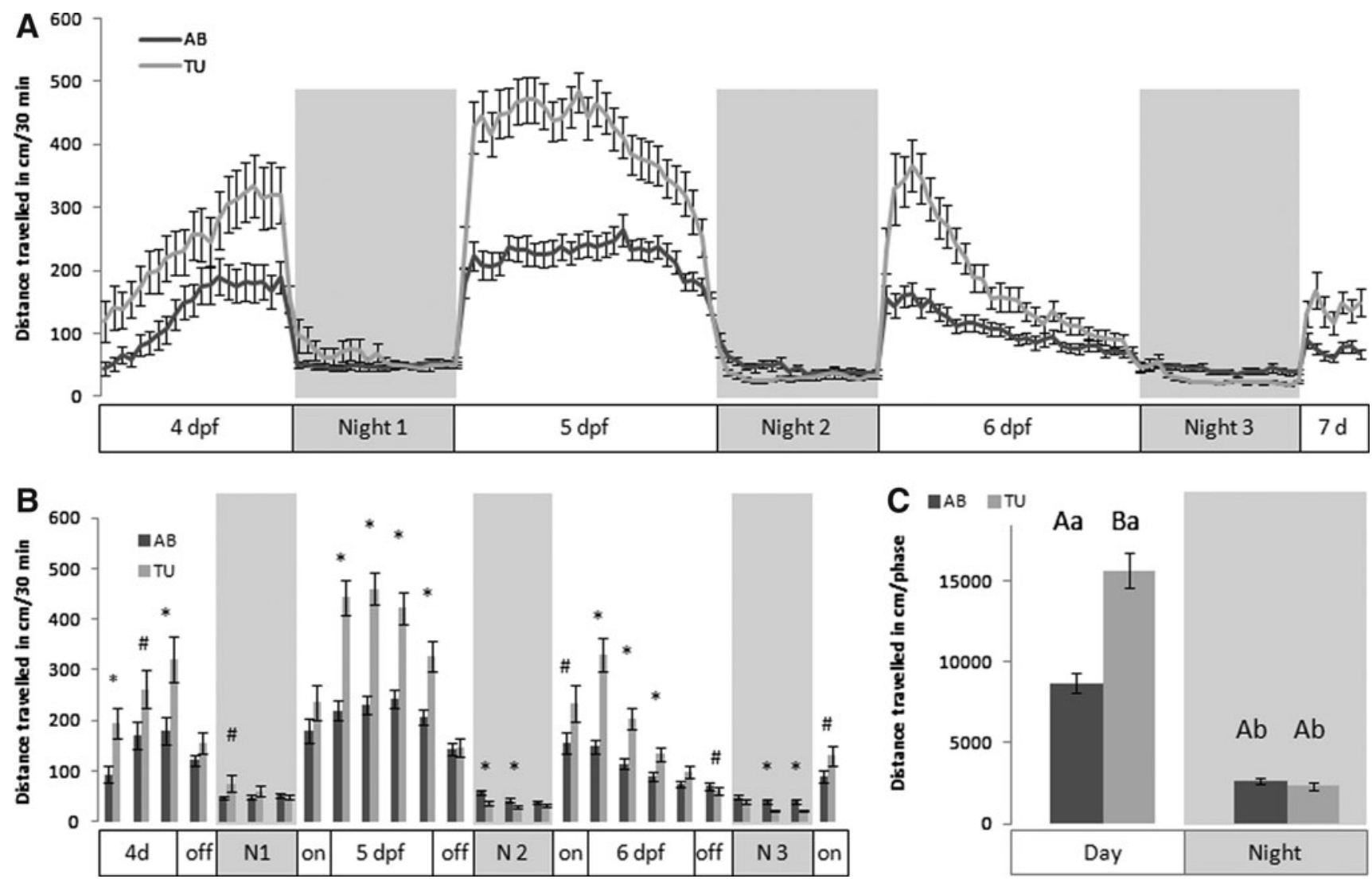

FIG. 2. Spontaneous activity of larvae over 72-h. (A) Locomotor activity nested per 30-min period. (B) Cumulated locomotor activity per block of 6-7 half hours. The grayscale background indicates the light status/period of the day. (C) Locomotor activity during daytime and nighttime periods. [Mean $\pm S E M$; ${ }^{*} p<0.05$; ${ }^{*} p<0.1$; in (C), upper case letters indicate significant differences between strains within periods and lower case letters indicate significant differences between periods within strains; $n=30$ per strain]. 


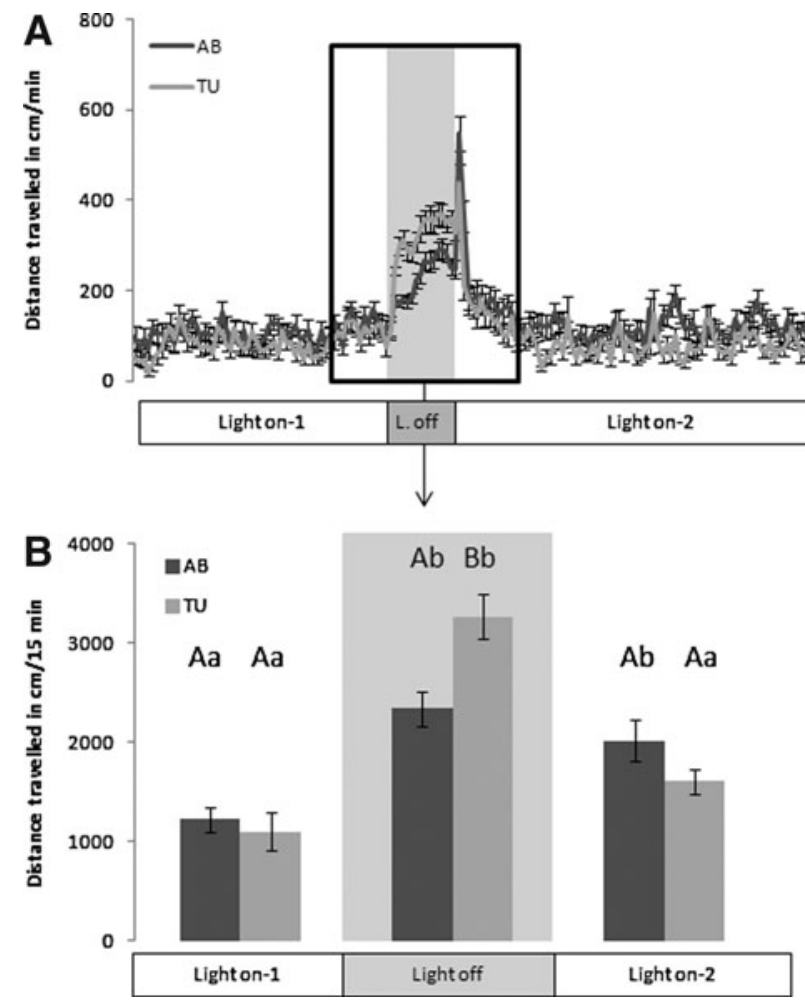

FIG. 3. Light/dark challenge with adult fish. (A) Locomotor activity was recorded for $1 \mathrm{~h}$ before a sudden dark period lasting $15 \mathrm{~min}$ (gray shadowing). The fish were recorded for the following $90 \mathrm{~min}$. (B) Locomotor activity during 15-min periods was used for statistical analyses. $[$ Mean $\pm S E M$; in (B), upper case letters indicate significant differences between strains within periods and lower case letters indicate significant differences between periods within strains; $n=18$ per strain].

showed that, within each period, there was no difference between TU and AB strains (Fig. 4B). Using a shorter alternative procedure, P2, dark challenge did not elicit any specific response although the subsequent light on-2 was associated with the classical decrease in activity (Fig. 5). Statistical analysis indicated that TU larvae were less active than $A B$ larvae during light on- 1 and light off periods, but not during light on-2 (RM-ANOVA $\mathrm{F}_{(3,200)}=6.52 ; p<0.001$; Fig. 5B).

\section{T-maze-exploration behavior and novel environment}

For this experiment with 12-month adults, mass were different $\left(\mathrm{AB}=409.0 \pm 24.0 \mathrm{mg}, \mathrm{TU}=343.7 \pm 16.9 \mathrm{mg}, \mathrm{F}_{(1,40)}=4.93\right.$, $p=0.032)$, but showed no interaction with strain, and length were homogeneous $(\mathrm{AB}=2.97 \pm 0.05 \mathrm{~cm}, \mathrm{TU}=2.88 \pm 0.03 \mathrm{~cm}$, $\left.\mathrm{F}_{(1,40)}=2.19, p=0.146\right)$. Similar numbers of fish of the two strains reached the deep area (18 and 16 of 20 for AB and TU strains, respectively), and the mean latency before reaching this zone was not significantly different (ANOVA $\left.\mathrm{F}_{(1,34)}=1.92 ; p=0.176\right)$. This is in agreement with the observation that the first exit from the start area did not differ between strains (ANOVA $\mathrm{F}_{(1,40)}=1.45 ; p=0.236$; Fig. 6A). Over the total duration of the challenge, TU fish tended to spend more time than $\mathrm{AB}$ fish in the start area (ANOVA $\left.\mathrm{F}_{(1,40)}=32.85 ; p=0.058\right)$, although the residence time in other

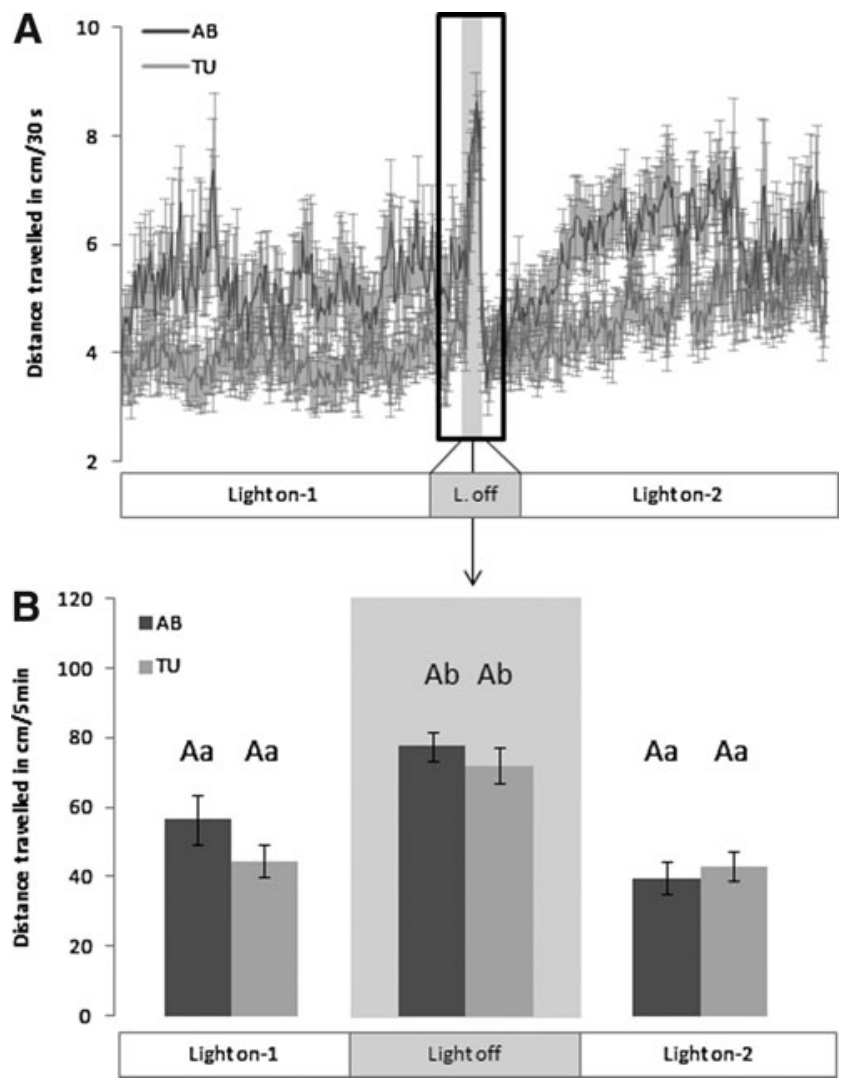

FIG. 4. Light/dark challenge with larvae-protocol P1. (A) Locomotor activity was recorded for the $2 \mathrm{~h}$ before a sudden dark period lasting $5 \mathrm{~min}$ (gray shadowing). The fish were recorded for a further $2 \mathrm{~h}$. (B) Locomotor activity during 5min periods was used for statistical analyses. [Mean \pm SEM; in (B), upper case letters indicate significant differences between strains within periods, and lower case letters indicate significant differences between periods within strains; $n=40$ per strain].

areas was not different (Fig. 6B). Monitoring of the locomotor activity in the shallow area showed a gradual increase of TU fish activity, which became significantly higher than $\mathrm{AB}$ fish activity from minute 3 (Fig. 6C; RM-ANOVA $\mathrm{F}_{(5,40)}=2.93$; $p=0.027$; Newman-Keuls post hoc test Min-1 $p=0.653$; Min-2 $p=0.397 ;$ Min-3 $p=0.024 ;$ Min-4 $p=0.003$; Min-5 $p=0.004$ ).

\section{Novel tank-comparison of homebase behavior}

For this experiment in 3-month old adults, both mass and length were different: $(\mathrm{AB}=145.1 \pm 17.6 \mathrm{mg}, \mathrm{TU}=285.6 \pm 31.4$ $\mathrm{mg}, \mathrm{F}_{(1,24)}=14.97, p<0.001 ; \mathrm{AB}=2.03 \pm 0.09 \mathrm{~cm}, \mathrm{TU}=2.45 \pm$ $\left.0.07 \mathrm{~cm}, \mathrm{~F}_{(1,24)}=14.35, p<0.001\right)$, but showed no interaction with strain. To compare anxiety levels between adults of the two strains, we used the novel tank diving test (Fig. 7A). Fish from both strains spent most of the time in the bottom zone of the tank, but in both cases, the time spent by fish in the top zone increased over time. Discretization of value per minute indicated that for most Min-periods, TU fish spent more time than $\mathrm{AB}$ fish in the top zone (Fig. 7B; RM-ANOVA $\mathrm{F}_{(6,24)}=1.7$; $p=0.165$; Newman-Keuls post hoc test Min-1 $p=0.018$; Min-2 $p=0.020$; Min-3 $p=0.105$; Min-4 $p=0.069$; Min-5 $p=0.020$; Min-6 $p=0.009)$. 

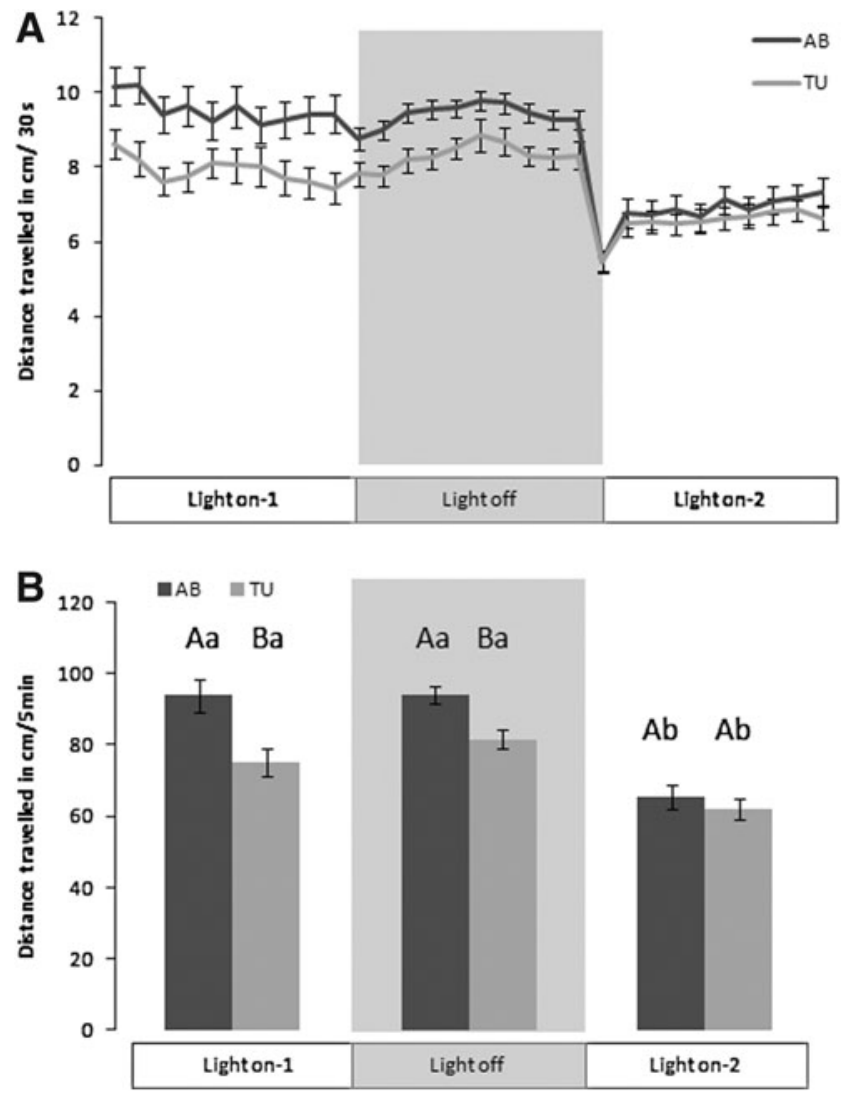

FIG. 5. Light/dark challenge with larvae-protocol P2. (A) Locomotor activity was recorded for $15 \mathrm{~min}$ consisting of light on/light off/light on periods, each of $5 \mathrm{~min}$. (B) Locomotor activity during 5-min periods was used for statistical analyses. [Mean \pm SEM; in (B), upper case letters indicate significant differences between strains within periods and lower case letters indicate significant differences between periods within strains; $n=100$ per strain].

\section{Color learning}

Fish were subjected to colored light conditioning during 15 days by associating feeding with artemias with a green light, as described in the Methods section and two color preference place tests were performed within the next 5 days to evaluate their learning abilities. In test 1 , conditioned fish of both strains spent significantly more time in the green chamber than in control chambers (ANOVA $\mathrm{F}_{(1,45)}=13.89 ; p<0.001$ for $\mathrm{AB}$ fish and $\mathrm{F}_{(1,45)}=4.89 ; p=0.032$ for TU fish; Fig. 8A). In the second test, designed to differentiate color conditioning from light conditioning, only conditioned fish were used. AB fish spent significantly more time in the green zone than in other zones (ANOVA $\mathrm{F}_{(2,30)}=6.16 ; p=0.003$ ), but this was not the case for TU fish (ANOVA $\left.\mathrm{F}_{(2,30)}=0.15 ; p=0.859\right)$ (Fig. 8B).

\section{Discussion}

The increasing use of zebrafish, and in particular for behavioral assays, in various fields of research, a detailed knowledge of elements, which can be a source of difference between replicates, is required. In addition, assays using behavioral endpoints relying on natural behavior are very diverse thereby increasing the difficulties of interpretation and comparison between studies. Several experiments using laboratory strains and WT populations have indicated that behavioral responses vary according to the strain used. Consequently, the fish strain could be an important source of variation between assays. We therefore conducted a systematic comparison of the behavior of two widely used laboratory WT strains: AB and TU.

We assessed the natural behavior of adults and larvae in the absence of challenges: we monitored the locomotor activity over long periods of time, including two or more night periods. Adults of both strains displayed U-shaped actograms, with the top of the $U$ corresponding to transition periods (between day and night and between night and day) and the bottom of the $U$ to the middle of the day and night periods. Discretization of the activity led to identification of differences between the two strains. Comparing daily and the nocturnal activity revealed a diurnal pattern for $A B$ fish, whereas the activity of TU fish was more evenly distributed. In the case of larvae, both strains were clearly diurnal with 5 to 10 times more activity during day periods than night periods over $72 \mathrm{~h}$ of recording. For both strains, there was a large increase in the activity during the fourth dpf. In the case of the $A B$ strain, activity plateaued from the end of $4 \mathrm{dpf}$, whereas for TU larvae, the increase continued until $5 \mathrm{dpf}$. This overall shape of the activity profile is consistent with the development of larvae standing on their side until $4 \mathrm{dpf}$, and then inflating their swim-bladder around $5 \mathrm{dpf}^{36}$ At this same stage, the larvae start feeding. A combination of swimming and feeding behaviors presumably, therefore, explains the surge of activity observed at 4-5dpf. This change in the daily activity has previously been described for WT populations. ${ }^{37}$ The development of locomotor behavior parallels the maturation of serotoninergic neurons, ${ }^{38}$ and the application of exogenous dopamine inhibits the initiation of swimming at $5 \mathrm{dpf}^{39} \mathrm{~A}$ large increase in the activity of larvae between 3 and $5 \mathrm{dpf}$ has also been reported by Thirumalai et al. ${ }^{39}$ but Prober et al. who monitored larvae from 5 to $7 \mathrm{dpf}$ found no such increase. ${ }^{40}$ Note, however, that Thirumalai et al. used 5-cm-diameter Petri dishes, whereas Prober et al. used 96-well plates, and this methodological difference can cause large differences in the behavior of larvae ${ }^{41}$; in addition, Prober et al. recorded activity as seconds of activity over $10 \mathrm{~min}$ rather than the true distance travelled. ${ }^{40}$ Prober et al. observed a decrease in activity by the end of $5 \mathrm{dpf}$ and during $6 \mathrm{dpf}$. This observation is in agreement with Experiment 2 reported by Colwill et al. ${ }^{37}$ and the findings by MacPhail et al. for the influence of experimental conditions on larval behavior at $6 \mathrm{dpf}^{9}$ MacPhail et al. also reported that the variability measured decreased along with the activity level during the day. These various observations were used to identify the afternoon, $6 \mathrm{dpf}$, as the preferred time period to perform individual larval behavioral assays. Similarly, we observed a stabilization of the activity level by the end of $6 \mathrm{dpf}$ as well as a reduction in variability. In MacPhail's study, there is no indication of the strain used. Note that we found TU to have a significantly higher diurnal activity than $\mathrm{AB}$. We are unaware of any previous demonstration of differences in the spontaneous locomotor activity between the larvae of different strains, although various short assays at different ages have been described. ${ }^{37,42}$

Long-duration assays are useful for analyzing the expression of natural patterns and divergence from them under experimental situations. However, they are not suitable for 


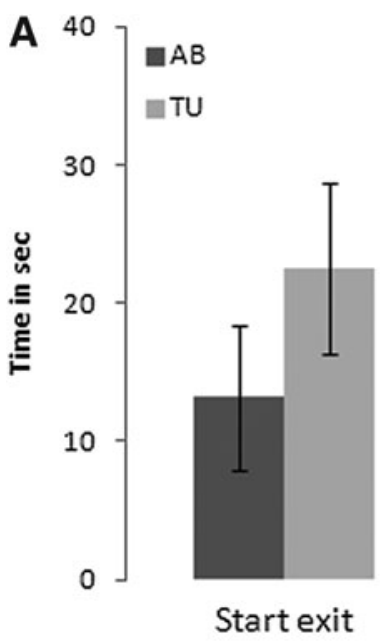

FIG. 6. Exploratory behavior in a T-maze device. (A) Time to first exit from the start zone. (B) Total time spent in T-maze areas (start, deep, and shallow). (C) Distance travelled nested in 1min bouts. (Mean \pm SEM; ${ }^{*} p<0.05 ;{ }^{\#} p<0.1 ; n=20$ per strain).
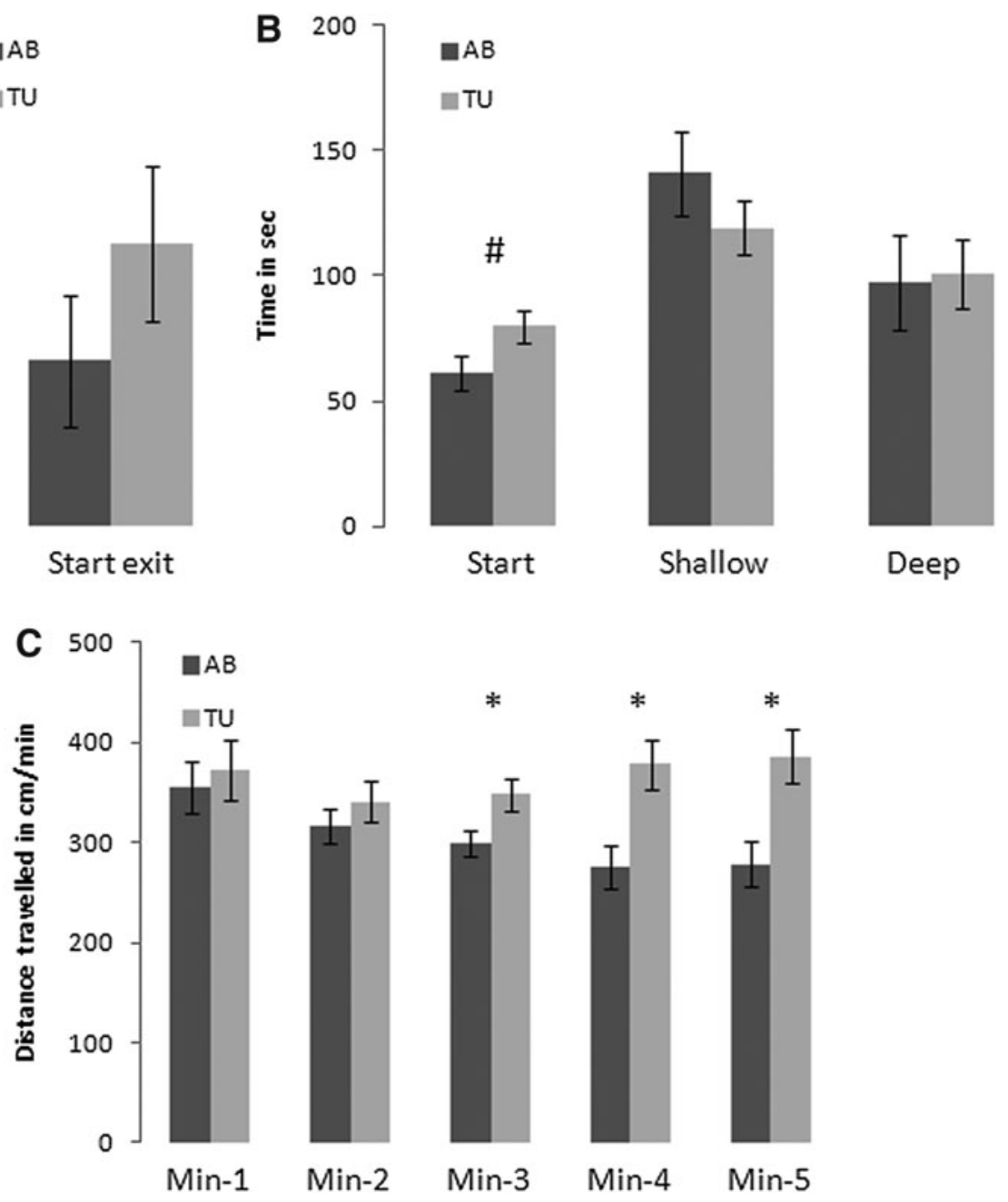

mass screening because they are mostly low throughput. Consequently, alternative, shorter procedures have been developed to trigger responses, and several such assays have been described for both larvae and older fish.

The light/dark challenge is straightforward, and is therefore widely used to trigger behavioral responses in larvae. We found that light transition also produced strong pulses of activity in adults, so we assessed the responses of fish at both stages. As expected, adults of both strains displayed a strong reaction to light changes, with a significant increase in activity during light-off. The TU strain displayed a larger response, with a threefold increase versus a twofold increase for AB fish. In the case of larvae, several protocols have been used with different step numbers and duration, with and without acclimatization, and different well sizes. ${ }^{41-43}$ We used two protocols, P1 with a 2-h acclimatization step inside the larvaebox and P2 without acclimatization. Using P1 protocol, we observed the expected increase in the larval activity associated with the light-off switch for both strains, and the expected subsequent decrease. These findings are in agreement with previously reported data ${ }^{9,41,42}$ and no differences were observed between strains. Because P1 only allowed assessment of 1 plate (20 larvae) per day, we also assessed P2, which allows a higher throughput. However, in P2 tests, and for both strains, the light-off switch did not elicit the expected increase in activity, although the following light-on induced the expected decrease in activity. $\mathrm{AB}$ larvae were more active than TU larvae during all three periods, the difference being particularly high for light on-1 and light off periods. Differences between laboratory strains in a similar assay have recently been reported: $5 \mathrm{dpf} A B$ larvae were more active than TL larvae during dark periods. We observed no change in the activity between light on- 1 and light off periods, and this is not consistent with previous reports for similar assays; this discrepancy is probably because in our protocol, activity monitoring started after an acclimatization period in the light as in the study by Ali et al. ${ }^{43}$ and not in the dark as was the case in several other studies. ${ }^{9,41,42}$ However, Ali and collaborators used 96-well plates, ${ }^{43}$ whereas we used 24-well plates, which have been shown to elicit a higher activity. ${ }^{41}$

Many tests have been developed to assess several adult behaviors in challenged situations. We chose to use wellestablished tests to assess responses to light stimuli and exploratory and novel environment behaviors. The T-maze device was used to assess exploration: other than TU fish spending more time in the start area, no differences were observed in the exploratory behavior between strains. The locomotor activity of TU fish significantly increased over the 5-min duration of the test. A parallel can be made with the vertical position in the novel tank test in which, TU fish displayed an early homebase 

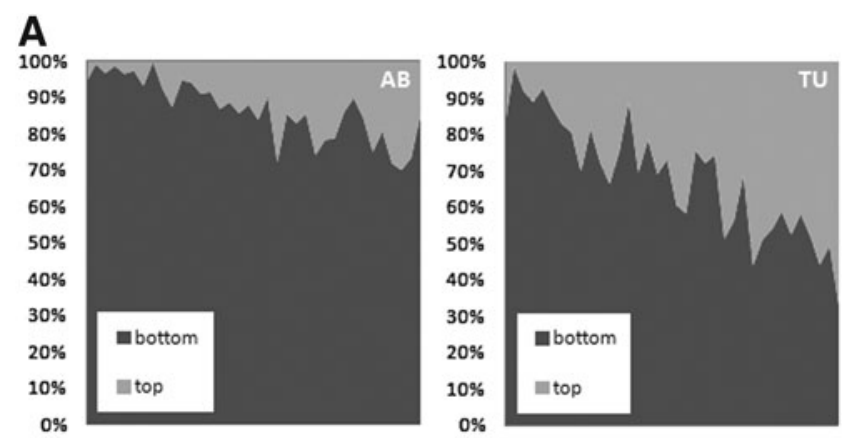

B

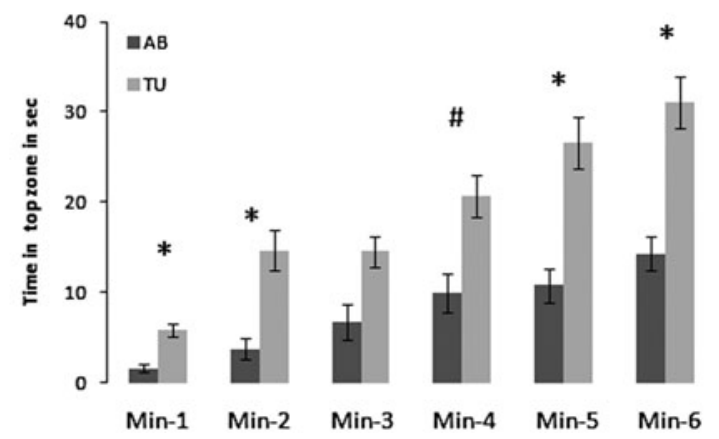

FIG. 7. Novel tank challenge. (A) Mean zone occupancy during $6 \mathrm{~min}$ for $\mathrm{AB}$ and $\mathrm{TU}$ fish. (B) Top zone residence time nested in 1-min bouts. (Mean \pm SEM; ${ }^{*} p<0.05 ;{ }^{\#} p<0.1 ; n=12$ per strain).

release with a significant increase in the time spent in the upper zone over the test duration and a significantly higher stay duration in the upper zone than $\mathrm{AB}$ fish. Homebase occupancy has been described to be an indicator of anxiety ${ }^{27,44,45}$ and we have also found an association between homebase release and hyperactive behavior. ${ }^{46}$

Our various findings suggest that adult $\mathrm{TU}$ fish were more active in a novel environment and that $A B$ fish displayed a more anxious behavior. Similar differences have been described in a report comparing a WT population with strains selected for particular phenotypes (albino, leopard, and longfin): commercial WT fish displayed less anxious behavior than mutant fish ${ }^{27}$; the authors pointed out that this behavioral difference should be taken into account when setting up experimental protocols and choosing strains. Our study extends this point to laboratory strains widely used in research. Our data suggest that to obtain the most significant results, the more anxious $\mathrm{AB}$ strain should be favored for evaluations of anxiolytic drugs/manipulations in adults, and that the TU strain should be favored for evaluations of anxiogenic drugs / manipulations. For investigations involving rhythm monitoring and/or long-term behavior in which the contrasted circadian activity is required, the $\mathrm{AB}$ strain should clearly be favored over the TU strain because it displays a robust diurnal rhythm. Finally, for assays involving color conditioning, the choice will depend on the complexity of tests: adult fish of both strains are efficiently conditioned by the green color as assessed by a green/no light shuttle box test. However, for three-color choice protocols (green/blue/no light), the $\mathrm{AB}$ strain may be more appropriate because they showed preference for the color used for conditioning, whereas TU fish did not. This difference in response to conditioning between $\mathrm{AB}$
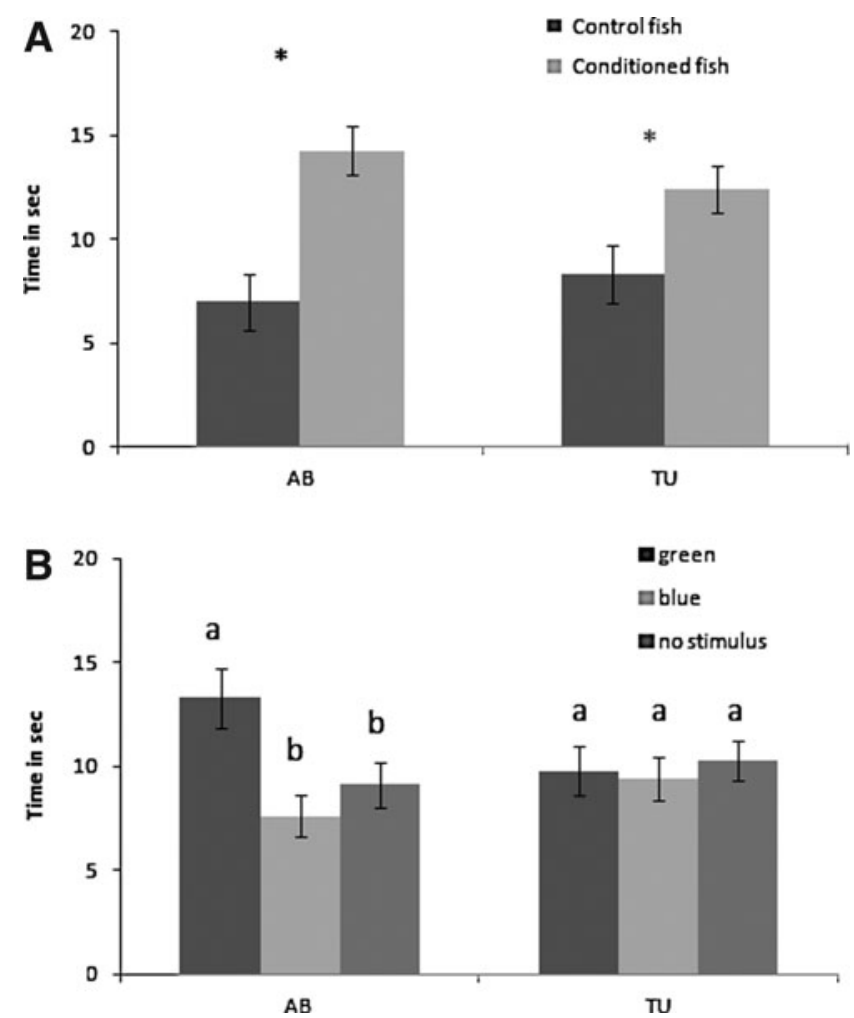

FIG. 8. Color conditioning. (A) Time spent in green chamber versus zones with no stimulus: the value is higher for conditioned fish than control fish for both strains (mean \pm SEM; ${ }^{*} p<0.05 ; n=15$ for control fish for both strains, $n=30$ for $\mathrm{AB}$ and TU conditioned fish). (B) Time spent in green chamber versus blue or no light chamber for $A B$ and TU conditioned fish (mean \pm SEM; different letters indicate significant differences between colors within strains, $n=30$ for each strain).

and TU strains is in agreement with the results of a conditioning preference place test. ${ }^{21}$

Several studies have reported behavioral differences between laboratory strains and commercial or wild-caught populations, ${ }^{17,18}$ including differences in behavioral activities, ${ }^{16,24,27,30}$ thigmotaxis, ${ }^{30}$ social behavior including (aggression, shoaling, and reproduction), 12,14,16,19-22,24,31,47 boldness with or without contamination $22,30,48,49$ and conditioning or learning new tasks. ${ }^{12,13,21,23}$ However, these studies comparing laboratory strains and WT populations clearly differ from ours, in which, two laboratory strains were compared. In addition, several previous studies used commercial populations, and their genetic background was not known. Nevertheless, they indicate that different genetic backgrounds can lead to behavioral differences, as already been demonstrated for other fish and other species. ${ }^{50,51}$ Laboratory strains have, since their establishment, been subject to classical selection based on zootechnical criteria, and in particular, robustness and fertility. Such directed selection processes are often accompanied by a relaxation of selection pressure on other previously essential criteria, for example, predator avoidance. This explains why independent domestication events may lead to convergent phenotypes for some criteria (e.g., robustness and fertility), but not others, like behavior (see ${ }^{24}$ and this work). 
In summary, using a large palette of behavioral monitoring methods, including long-term and challenge assays, we demonstrate that zebrafish strains $\mathrm{AB}$ and $\mathrm{TU}$, widely used in research, display some similar and some divergent behaviors. Similar and different behaviors were observed for both larval and juvenile/adult stages. Since zebrafish behavioral tests, including simple monitoring of the locomotor activity of larvae, are being increasingly used in diverse disciplines, care should be taken to select appropriate strains, and in particular for work involving large-scale screening. ${ }^{52-54}$ The recent report that maturation of shoaling differs between $\mathrm{AB}$ and $\mathrm{TU}$ strains and is associated to differences in dopamine and serotonin levels in the brain of developing fish shed light on possible mechanisms underlying such interstrain behavioral differences. $^{31}$

\section{Acknowledgments}

Thanks to Kaspar Müller and Stefan Neuhauss for sharing unpublished data on fish color preference. This work was performed with the financial support Agence Nationale pour la Recherche [CES-2009-002 ConPhyPoP to X.C.]. Funds from the Région Poitou-Charentes and from l'Institut Français de Recherche pour l'Exploitation de la Mer (Ifremer) supported C.V. and S.P. during their PhD researches.

\section{Disclosure Statement}

There are no competing financial interests.

\section{References}

1. Steenbergen PJ, Richardson MK, Champagne DL. The use of the zebrafish model in stress research. Prog Neuropsychopharmacol Biol Psychiatry 2011;35:1432-1451.

2. Champagne DL, Hoefnagels CCM, de Kloet E, Richardson K. Translating rodent behavioral repertoire to zebrafish (Danio rerio): Relevance for stress research. Behav Brain Res 2010;214:332-342.

3. Creton R. Automated analysis of behavior in zebrafish larvae. Behav Brain Res 2009;203:127-136.

4. Norton W, Bally-Cuif L. Adult zebrafish as a model organism for behavioural genetics. BMC Neurosci 2010;11:90.

5. Hill AJ, Teraoka H, Heideman W, Peterson RE. Zebrafish as a model vertebrate for investigating chemical toxicity. Toxicol Sci 2005;86:6-19.

6. Hinton DE, Kullman SW, Hardman RC, Volz DC, Chen P-J, Carney $\mathrm{M}$, et al.: Resolving mechanisms of toxicity while pursuing ecotoxicological relevance? Mar Pollut Bull 2005;51:635-648.

7. Spitsbergen JM, Kent ML. The State of the art of the zebrafish model for toxicology and toxicologic pathology researchadvantages and current limitations. Toxicol Pathol 2003;31: 62-87.

8. Linney E, Upchurch L, Donerly S. Zebrafish as a neurotoxicological model. Neurotoxicol Teratol 2004;26:709-718.

9. MacPhail RC, Brooks J, Hunter DL, Padnos B, Irons TD, Padilla S. Locomotion in larval zebrafish: influence of time of day, lighting and ethanol. NeuroToxicology 2009;30:52-58.

10. Blechinger SR, Kusch RC, Haugo K, Matz C, Chivers DP, Krone $\mathrm{PH}$. Brief embryonic cadmium exposure induces a stress response and cell death in the developing olfactory system followed by long-term olfactory deficits in juvenile zebrafish. Toxicol Appl Pharmacol 2007;224:72-80.
11. Scherer E. Behavioural responses as indicators of environmental alterations: approaches, results, developments. Appl Ichthyol 1992;8:122-131.

12. Zala SM, Määttänen I, Penn DJ. Different social-learning strategies in wild and domesticated zebrafish, Danio rerio. Anim Behav 2012;83:1519-1525.

13. Spence R, Magurran AE, Smith C. Spatial cognition in zebrafish: the role of strain and rearing environment. Anim Cogn 2011;14:607-612.

14. Benner MJ, Drew RE, Hardy RW, Robison BD. Zebrafish (Danio rerio) vary by strain and sex in their behavioral and transcriptional responses to selenium supplementation. Comp Biochem Physiol A Mol Integr Physiol 2010;157:310318.

15. Drew RE, Settles ML, Churchill EJ, Williams SM, Balli S, Robison BD. Brain transcriptome variation among behaviorally distinct strains of zebrafish (Danio rerio). BMC Genomics 2012;13:323.

16. Moretz J, Martins E, Robison B. The effects of early and adult social environment on zebrafish (Danio rerio) behavior. Environ Biol Fish 2007;80:91-101.

17. Oswald M, Robison BD. Strain-specific alteration of zebrafish feeding behavior in response to aversive stimuli. Can J Zool 2008;86:1085-1094.

18. Robison BD, Rowland W. A potential model system for studying the genetics of domestication: behavioral variation among wild and domesticated strains of zebra danio (Danio rerio). Can J Fish Aquat Sci 2005;62:2046-2054.

19. Soffker M, Stevens JR, Tyler CR. Comparative breeding and behavioral responses to ethinylestradiol exposure in wild and laboratory maintained zebrafish (Danio rerio) populations. Environ Sci Technol 2012;46:11377-11383.

20. Barba-Escobedo PA, Gould GG. Visual social preferences of lone zebrafish in a novel environment: strain and anxiolytic effects. Genes Brain Behav 2012;11:366-373.

21. Ninkovic J, Bally-Cuif L. The zebrafish as a model system for assessing the reinforcing properties of drugs of abuse. Methods 2006;39:262-274.

22. Dlugos CA, Rabin RA. Ethanol effects on three strains of zebrafish: model system for genetic investigations. Pharmacol Biochem Behav 2003;74:471-480.

23. Vital C, Martins EP. Strain differences in zebrafish (Danio rerio) social roles and their impact on group task performance. J Comp Psychol 2011;125:278-285.

24. Moretz JA, Martins EP, Robison BD. Behavioral syndromes and the evolution of correlated behavior in zebrafish. Behav Ecol 2007;18:556-562.

25. Scerbina T, Chatterjee D, Gerlai R. Dopamine receptor antagonism disrupts social preference in zebrafish: a strain comparison study. Amino Acids 2012;43:2059-2072.

26. Sackerman J, Donegan JJ, Cunningham CS, Nguyen NN, Lawless K, Long A, et al. Zebrafish behavior in novel environments: effects of acute exposure to anxiolytic compounds and choice of Danio rerio line. Int J Comp Psychol 2010;23: 43-61.

27. Egan RJ, Bergner CL, Hart PC, Cachat JM, Canavello PR, Elegante $\mathrm{MF}$, et al. Understanding behavioral and physiological phenotypes of stress and anxiety in zebrafish. Behav Brain Res 2009;205:38-44.

28. Winter MJ, Redfern WS, Hayfield AJ, Owen SF, Valentin J-P, Hutchinson TH. Validation of a larval zebrafish locomotor assay for assessing the seizure liability of early-stage development drugs. J Pharmacol Toxicol Methods 2008;57: 176-187. 
29. Coe TS, Hamilton PB, Griffiths AM, Hodgson DJ, Wahab MA, Tyler CR. Genetic variation in strains of zebrafish (Danio rerio) and the implications for ecotoxicology studies. Ecotoxicology 2009;18:144-150.

30. Lockwood B, Bjerke S, Kobayashi K, Guo S. Acute effects of alcohol on larval zebrafish: a genetic system for largescale screening. Pharmacol Biochem Behav 2004;77:647654.

31. Mahabir S, Chatterjee D, Buske C, Gerlai R. Maturation of shoaling in two zebrafish strains: a behavioral and neurochemical analysis. Behav Brain Res 2013;247:1-8.

32. Cousin X, Daouk T, Péan S, Lyphout L, Schwartz M-E, Bégout M-L. Electronic individual identification of zebrafish using radio frequency identification (RFID) microtags. J Exp Biol 2012;215:2729-2734.

33. Blaser R, Gerlai R. Behavioral phenotyping in zebrafish: Comparison of three behavioral quantification methods. Behav Res Methods 2006;38:456-469.

34. Risner ML, Lemerise E, Vukmanic EV, Moore A. Behavioral spectral sensitivity of the zebrafish (Danio rerio). Vis Res 2006;46:2625-2635.

35. Mueller KP, Neuhauss SC. Automated visual choice discrimination learning in zebrafish (Danio rerio). J Integr Neurosci 2012;11:73-85.

36. Goolish EM, Okutake K. Lack of gas bladder inflation by the larvae of zebrafish in the absence of an air-water interface. J Fish Biol 1999;55:1054-1063.

37. Colwill RM, Creton R. Locomotor behaviors in zebrafish (Danio rerio) larvae. Behav Processes 2011;86:222-229.

38. Brustein E, Saint-Amant L, Buss RR, Chong M, McDearmid JR, Drapeau P. Steps during the development of the zebrafish locomotor network. J Physiol Paris 2003;97:77-86.

39. Thirumalai V, Cline HT. Endogenous dopamine suppresses initiation of swimming in prefeeding zebrafish larvae. J Neurophysiol 2008;100:1635-1648.

40. Prober DA, Rihel J, Onah AA, Sung R-J, Schier AF. Hypocretin/orexin overexpression induces an insomnia-like phenotype in zebrafish. J Neuroscience 2006;26:13400-13410.

41. Padilla S, Hunter DL, Padnos B, Frady S, MacPhail RC. Assessing locomotor activity in larval zebrafish: Influence of extrinsic and intrinsic variables. Neurotoxicol Teratol 2011;33:624-630.

42. de Esch C, van der Linde H, Slieker R, Willemsen R, Wolterbeek A, Woutersen $\mathrm{R}$, et al. Locomotor activity assay in zebrafish larvae: Influence of age, strain and ethanol. Neurotoxicol Teratol 2012;34:425-433.

43. Ali S, Champagne DL, Alia A, Richardson MK. Large-scale analysis of acute ethanol exposure in zebrafish development: a critical time window and resilience. PLoS One 2011; 6:e20037.

44. Stewart A, Cachat J, Wong K, Gaikwad S, Gilder T, DiLeo J, et al. Homebase behavior of zebrafish in novelty-based paradigms. Behav Processes 2010;85:198-203.

45. Stewart A, Gaikwad S, Kyzar E, Green J, Roth A, Kalueff AV. Modeling anxiety using adult zebrafish: a conceptual review. Neuropharmacology 2012;62:135-143.

46. Péan S. Caractérisation des altérations natatoires, alimentaires et sociaux des poissons en réponse aux polluant organiques persistants. Ed. Université de La Rochelle, France. p. 246, 2012.

47. Maack G, Segner H, Tyler CR. Ontogeny of sexual differentiation in different strains of zebrafish (Danio rerio). Fish Physiol Biochem 2003;28:125-128.

48. Loucks E, Carvan III MJ. Strain-dependent effects of developmental ethanol exposure in zebrafish. Neurotoxicol Teratol 2004;26:745-755.

49. Wright D, Rimmer LB, Pritchard VL, Krause J, Butlin RK. Inter and intra-population variation in shoaling and boldness in the zebrafish (Danio rerio). Naturwissenschaften 2003;90:374-377.

50. Wright D, Nakamichi R, Krause J, Butlin RK. QTL analysis of behavioral and morphological differentiation between wild and laboratory zebrafish (Danio rerio). Behav Genet 2006;36:271-284.

51. Kopp C. Locomotor activity rhythm in inbred strains of mice: implications for behavioural studies. Behav Brain Res 2001;125:93-96.

52. Kokel D, Peterson RT. Chemobehavioural phenomics and behaviour-based psychiatric drug discovery in the zebrafish. Brief Funct Genomics Proteomics 2008;7:483-490.

53. Kokel D, Peterson RT. Using the zebrafish photomotor response for psychotropic drug screening. Methods Cell Biol 2011;105:517-524.

54. Rihel J, Schier AF. Behavioral screening for neuroactive drugs in zebrafish. Dev Neurobiol 2012;72:373-385.

Address correspondence to: Xavier Cousin, PhD

Laboratoire d'Ecotoxicologie Ifremer

Place Gaby Coll BP 7

17137 L'Houmeau

France

E-mail: xavier.cousin@ifremer.fr 